\title{
DESIGN OF ADAPTIVE FUZZY TRACKING CONTROLLER FOR ROBOT NAVIGATION USING PARTICLE SWARM OPTIMIZATION
}

\author{
Pallavi P. Lingawar*1, K.K. Jajulwar ${ }^{2}$ \\ ${ }^{*}$ Dept of Communication Engineering, G.H.Raisoni College of Engineering \& Technology, Nagpur, India Email: \\ pplingawar@gmail.com; \\ ${ }^{2}$ Dept of Communication Engineering, G.H.Raisoni College of Engineering \&Technology, Nagpur, India Email: \\ Kapil.jajulwar@raisoni.net;
}

\section{*Corresponding Author: -}

Email: pplingawar@gmail.com;

\begin{abstract}
: -
This paper proposes a methodology for mobile robot navigation using the concept of Fuzzy Tracking Controller. The objective is to Design adaptive fuzzy controller for $x$-direction and y-direction movement. Mostly it is used in Robot Localization, Obstacle avoidance, Mapping, recognizing people and objects \&learn how to interact (e.g., grasp) with objects in Robot Navigation. It determines the safe path for the robot to transverse to its target location, while avoiding obstacles along the way. In this System it calculates the reference path and using Particle Swarm Optimization technique it will reduce Time required to reach the target. Fuzzy tracking controller will be used to direct the system to the destination which will help system to overcome the obstacles in the path and reach the accurate destination.
\end{abstract}

Keywords: - PSO (Particle Swarm Optimization), fuzzy logic controller, Navigation.

\section{(c) $(\$)$}




\section{INTRODUCTION}

Navigation is a field of study that focuses on the process of monitoring and controlling the movement of a craft or vehicle from one place to another. The field of navigation includes the four basics categories such as aeronautic navigation, marine navigation, land Navigation and space navigation. Locating the Navigator's position compared to known locations or patterns includes in navigational techniques. Robot navigation means the robot's ability to determine its own position in its frame of reference and then to plan a path towards some goal location. Navigation can be defined as the combination of the three fundamental competences: Self-localization, Path planning, Map-building and map interpretation

Robot navigation has long been a fundamental goal in both computer vision research and robotics. While the problem is largely solved for robots equipped with active range-finding devices, for a variety of reasons, the task still remains challenging for robots equipped only with vision sensors. Vision is an attractive sensor as it helps in the design of economically viable systems with simpler sensor limitations. Sensors like ultrasonic, IR, GPS, laser sensors etc are used in conventional robot navigation systems, utilizing traditional suffer several drawbacks related to either the physical limitations of the sensor. Vision sensing has emerged as a popular alternative where cameras can be used to minimize the overall cost and maintaining high degree of intelligence, robustness and flexibility.

"The world is only shades of gray but not black and white. "Zadeh introduced fuzzy sets in 1965, sets with unsharp boundaries. Rather than black and white These sets are considered gray are a sin contrast to classical sets which form the basis of binary or Boolean logic.

In this paper, basic concepts of fuzzy logic are briefly presented. Its utility for synthesis of control systems is discussed in the context of an application to mobile robot motion control. In mobile robotics, a fuzzy logic-based control system has the advantage that it allows the intuitive nature of collision-free navigation to be easily modeled using linguistic terminology. Due to the relative computational simplicity of fuzzy rule-based systems, intelligent decisions can be made in real-time, thus allowing for uninterrupted robot motion. Moreover, accurate (expensive) sensors and detailed models of the environment are not absolutely necessary for autonomous navigation.

\section{RELATED WORK}

In some literature certain factor were taken in to consideration GA based \& Particle Swarm Optimization based design approaches and two variants of hybrid design approaches for optimal controller design have been implemented for robot navigation in unknown environments. Particle Swarm Optimization based hybrid methodology is considered to be superior approach for this study.

Literature [1] proposes a method for vision based mobile robot navigation which uses stable fuzzy tracking controller design which is based on lyapunov theory and it also uses Particle Swarm Optimization hybrid methodology. Work has been done on two factors:

1. By using single camera-based vision sensor which captures images periodically.

2. Secondly it utilizes the method path planning to investigate reference path.

Literature [2] proposes scheme of situating mobile robots to move around in large complex or building for criminal or theft location detection prompt for some time. This study approaches to a mathematical model which helps in development of a scheme for an autonomous low-cost mobile robot system using visual SLAM (simultaneous localization \& mapping) $\&$ Particle Swarm Optimization planner. The study could provide a cost-effective solution for problem in indoor mobile robot navigation.

The EKF (extended Kalman filter) is well known choice for localization when a prior knowledge is well known. Literature [3] proposes develop adaptive Neuro-Fuzzy inference system for localization based EKF when a priori knowledge is incorrect. The propose system aims to start the system with incorrect knowledge of statistics on the basis of adaptive Neuro-Fuzzy inference system which tries to minimize the mis-matching between theoretical and actual values. this method is beneficial because of consistency in this approach is more than localization based EKF.

Literature [4] uses PID controller for autonomous mobile robot Navigation was implemented in PID controller using FPGA card. The PID controller was first simulated in simulink Then FPGA card was used to construct the PID controller in combination with stamp micro controller. Finally, performance of PID controller used to navigate robot.

\section{III.PROBLEM DEFINATION}

To search for an optimal path successfully, the model of an environment should be constructed first as shown in fig1. Environment Fig.1 shows the environment. It is a two-dimensional rectangular workspace where all the objects including robot, obstacles, source and destination point are locatedMobile Robotis defined as a Green square object which is represented by $C(C x, C y)$ in the $2 \mathrm{D}$ space.

Obstaclesare unpredictable objects that the robot may encounter during the execution of the task. In general, obstacles can be of any shape and size with a representation of each point by $O(O x, O y)$.To avoid a safe region around obstacles, the obstacle is wrapped by a circle. The radius, $\mathrm{R}$ of the circle is chosen in accordance with the size of the obstacle Goalis defined as a Red square object which is represented by $G(G x, G y)$ in the 2D space 


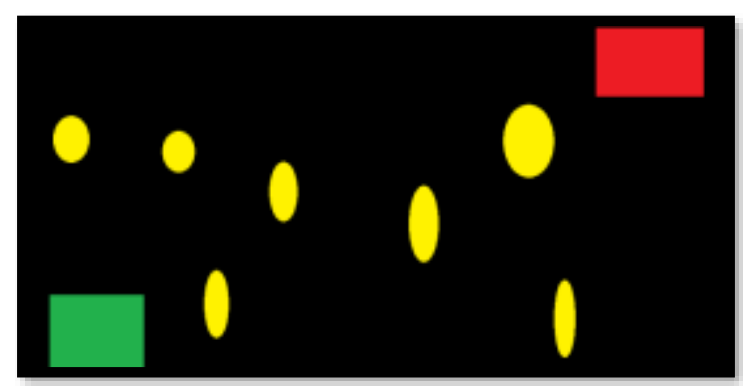

Figure:1 Indoor Environment for Mobile robot Navigation

\section{A. Assumption}

- The obstacles are detected by the Adaptive fuzzy Tracking controller So, the obstacles within the range, can be detected with reference to the coordinate system.

- The obstacles can be overlapping.

- The path planning program run until the goal has been returned.

\section{B. Input}

- Initial location of robot (Red square in bottom-left).

- Location of Destination (Green square in Fig. 1) and

- Position of the obstacles detected by sensor. (Cyan circles)

\section{Outcome of each step}

Next step of robot towards the feasible path by avoiding obstacle/s.

\section{Final Output}

A shortest pathway which is not crashed with the given obstacle/s.

\section{IV.PROPOSED METHODOLOGY}

Consider images stored in Database. One of the images is consider from the Database. It Is Difficult to Controller work on RGB level. Therefore, it is needed to convert in gray level image. Then that gray image is fed to controller. Working of controller as shown in below Flowchart. Robot is considered as X. After the controller find out destination then PSO will be applied on it to minimize the time delay.

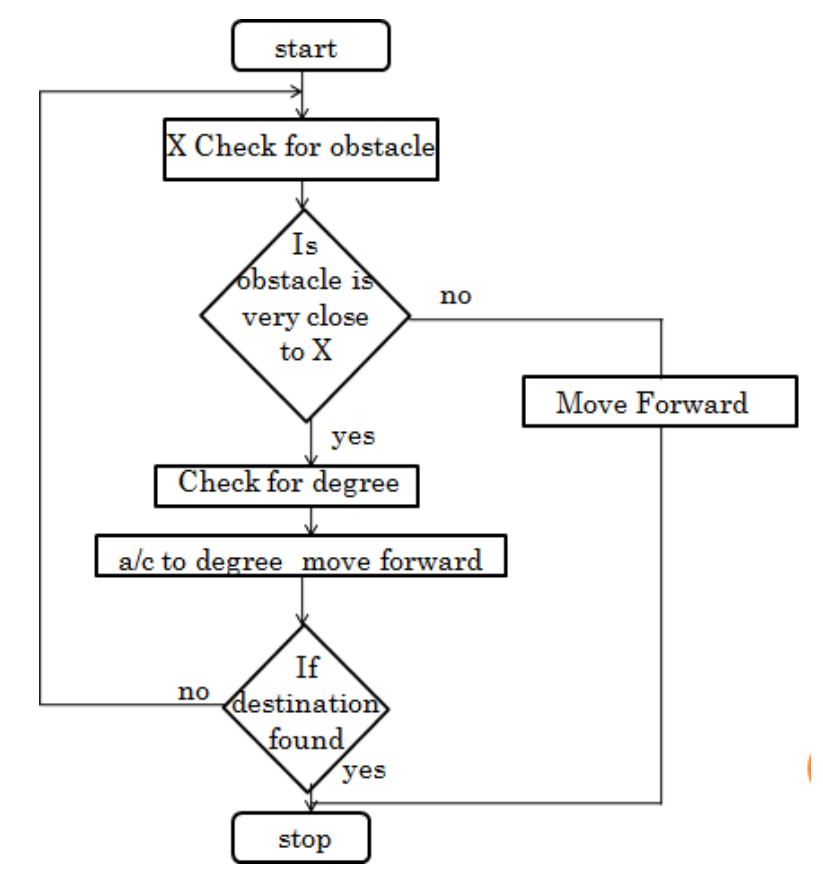

FIGURE:2 FLOW CHART OF ADAPTIVE FUZZY CONTROLLER

\section{APPROACH}

\section{A. Adaptive Fuzzy Tracking Controller}

Fuzzy logic-based controllers are expert control systems that smoothly interpolate between rules. Rules fire to continuous degrees and the multiple resultant actions are combined into an interpolated result Processing of uncertain information 
and savings of energy using common-sense rules and natural language statements are the bases for fuzzy logic control. Fuzzy controller rule-bases typically take the form of a set of if-then rules whose antecedents ("if" parts) and consequents ("then" parts) are propositions involving fuzzy membership functions. If X and Y are input and output universes of discourse of a fuzzy controller with a rule-base of size $n$, the usual if-then rule takes the following form IF $x$ is $\sim$ A $i$ THEN $y$ is ${ }^{\sim} B$

Where, $x$ and $y$ represent input and output fuzzy linguistic variables, respectively, and ${ }^{\sim} \mathrm{A} i € \mathrm{X}$ and ${ }^{\sim} \mathrm{B} i € \mathrm{Y}(1 \leq i \leq n)$ are fuzzy sets representing linguistic values of $x$ and $y$.

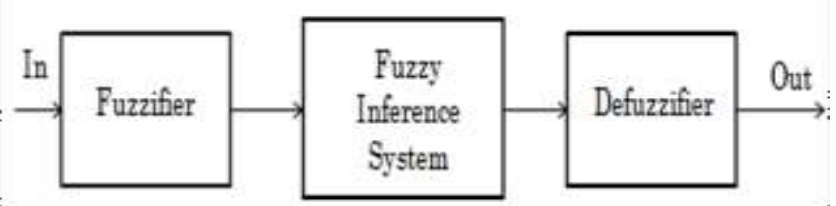

Figure:3 Fuzzy Logic Controller

Our purpose is to design a FLC able to guide a robot in its navigation in an environment from a start position to a goal zone. So, for this reason we adopted the following fuzzy logic system: In the Fuzzification, the inputs variables of our FLC are dl, dc, dr, dLr, dLl and _; where: dl, dc and dr: are respectively the distances to the nearest obstacles in the left, the center and the right sides of the robot, dLr, dLl: are respectively the distances to the nearest obstacles in the lateral right and lateral left sides of the robot and a: is the angle value between the robot direction and the target position. The variation range of variables $\mathrm{dl}$, $\mathrm{dc}$ and $\mathrm{dr}$ is $[0,2000]$ in millimeters; $0 \mathrm{~mm}$ means that the obstacle is very near and a collision may be happened, $2000 \mathrm{~mm}$ means that the obstacle is very far. Each of those three variables are represented by two trapezoidal Mfs: Near and Far. Whereas values of $\mathrm{dLr}$ and $\mathrm{dLl}$ are either 0 or 1 depending on the presence of obstacle or not. While the variation range of the input variable a is $\left[-180^{\circ}, 180^{\circ}\right]$ in which negative values refer to the left side robot and positive ones to the right side. Concerning the output variable of our FFLC we defined the angular velocity computed from the left and right robot's angular velocities values Vangular; the robot is supposed to have a fixed linear velocity equal to $100 \mathrm{~mm} / \mathrm{s}$. The range of variation of the output is $[-40,40]$; it is represented by five singleton MFs: T.Left; to orders the robot to turn to its left side, T.L.left to turn little left, Zero to forward, T.L.Right to turn little to its right side and right to make the robot turning to the right side. Regarding the inference part, in our controller we elaborated rules in such a way the robot behavior mimics the human comportment in driving.

\section{B. Particle Swarm Optimization}

Particle Swarm Optimization (PSO) was introduced by Eberhart and Kennedy in 1995. This technique was inspired by bird flocking's social behavior. Where it was observed that the motion of each bird is influenced by the motion of the nearest bird to it. PSO was used successfully in several applications. The algorithm initializes the population with random solutions and updates the generation to found an optima. A swarm consists of $\mathrm{N}$ particles in a $\mathrm{M}$-dimensional search space. In PSO each individual or particle has a position and a velocity expressed by a position change which means the flying direction of the particle. It successively adjusts its position based on two factors: the best position of its neighbors (pbest) and the best position visited by the whole swarm (gbest). The best solution is determined using a defined fitness function. Each particle is represented by its instantaneous current position $\mathrm{x}$ and position change _(x) or also named velocity, which are generally calculated as below:

$\mathrm{v}[]=\mathrm{c} 0 * \mathrm{v}[]+\mathrm{c} 1 * \operatorname{rand}() *($ pbest[] - present[] $)+\mathrm{c} 2 * \operatorname{rand}() *($ gbest[] - present $)$

$\&$

present[] $=$ present[] $+\mathrm{v}[]$

Where $\mathrm{w}$ indicates the inertia weight, $\mathrm{t}$ is the $\mathrm{t}^{\text {th }}$ iteration, rand() is a random function in [0 1], gbest is the global best of the whole swarm, pbesti is the local best of the $\mathrm{i}^{\text {th }}$ particle and $\mathrm{fc} 1$; $\mathrm{c} 2 \mathrm{~g}$ are cognitive and social parameters. The swarm size is assumed to be D.

In the algorithm the position change of each particle is limited by a maximum value _(x)max.

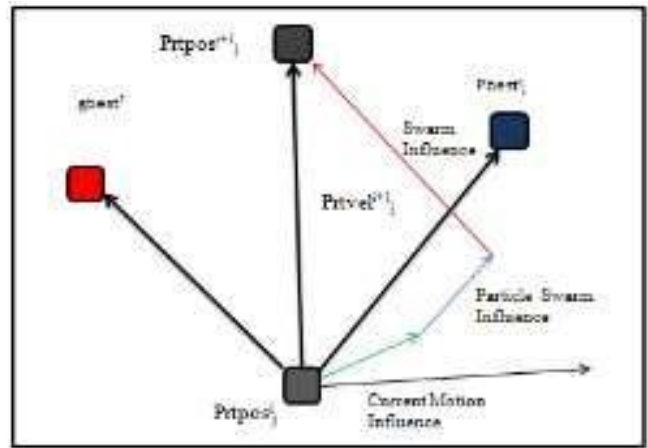

Figure:4 Description of particle's position in PSO 
The overall steps of the algorithm are as follow:

Step1: Initialization of the swarm.

Step2: Evaluation of the fitness value for each particle, evaluation of the particle's best position and the global best position of the swarm. Updating the particle's velocity and position using equation.

Step3: If one of the stopping criteria is achieved, then stop, or else go to step 2. Step4: Optimal solution (latest gbest) is generated.

Fig.4 illustrates the schematic view of updating position of particle in two successive iterations.

\section{VI.SIMULATION RESULT}

The starting direction is found from the start point and the end point. The inputs are three sensors for detection of obstacle which fuzzified on the basis of far, close or very close and the output is the navigation i.e. straight, soft right, hard right, soft left, hard left. The distance of the obstacle is sensed from the sensor and depends on the current position and orientation of the moving robot.

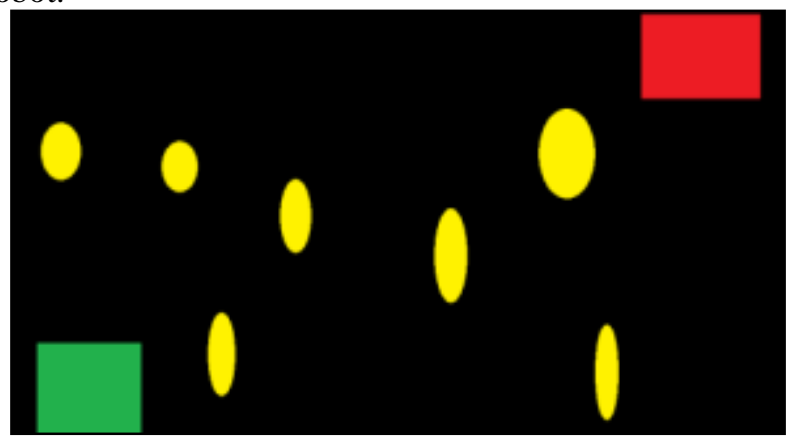

Figure:5 Indoor Environment consist of source and destination with obstacle

The environment where controller work shown in following figure. Figure:5 consist of source goal along with obstacle, Figure: 6 Shows possible path here only three possible paths considered out of which one is shortest one shown in

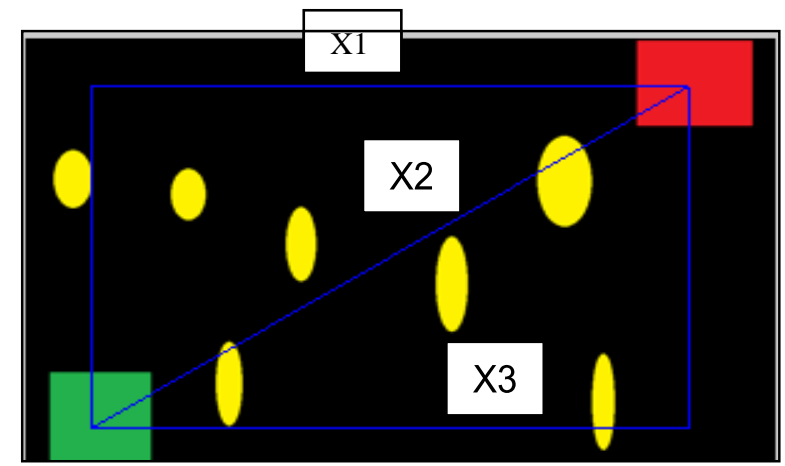

Figure:6 Indoor Environment Possible paths

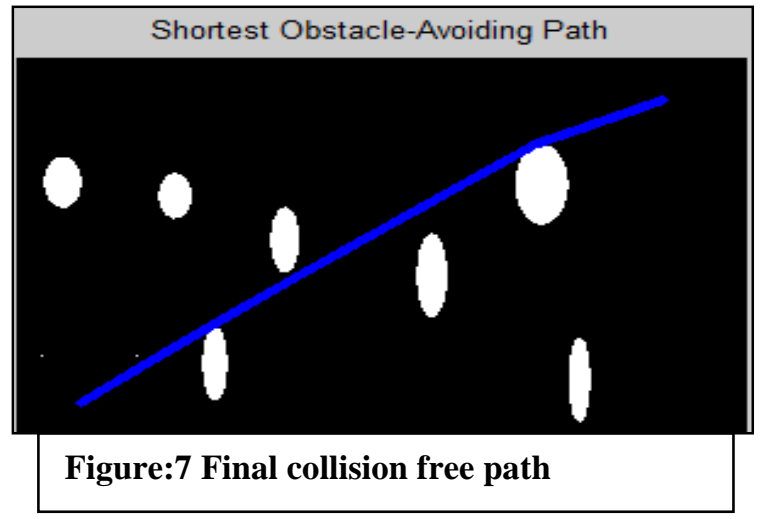

Table:1

\begin{tabular}{|l|c|c|}
\hline $\begin{array}{l}\text { Sr. } \\
\text { No. }\end{array}$ & $\begin{array}{c}\text { Indoor Environment } \\
\text { Possible Path }\end{array}$ & $\begin{array}{l}\text { No. Of Obstacle } \\
\text { in Given path }\end{array}$ \\
\hline 1 & $\mathrm{X} 1$ & 1 \\
\hline 2 & $\mathrm{X} 2$ & 2 \\
\hline 3 & $\mathrm{X} 3$ & 1 \\
\hline
\end{tabular}


Table:1 shows the Possible Path and Path with No. of obstacle. Whereas table2 Shows Movement of Robot and Obstacle Position.

Here Distance between Sourceand Destination considered 10 units then it is divided into 3 parts according to the Ranges As for very close, close and far. Three sensor are considered in Left, Right and Straight direction respectively. Depending upon the distance and direction membership function has been calculated.

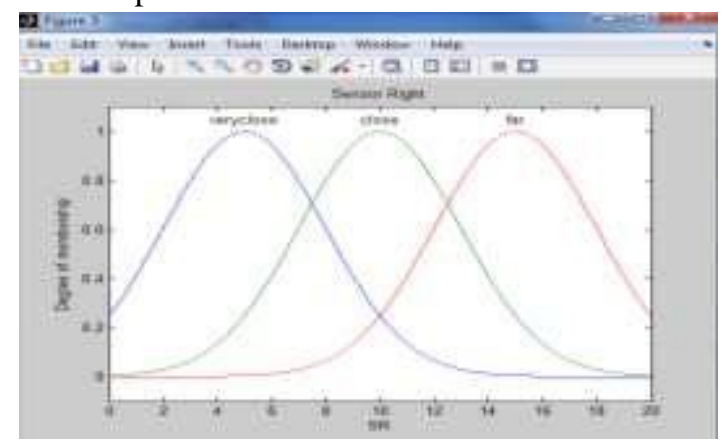

Table:2 Position of Right Sensor

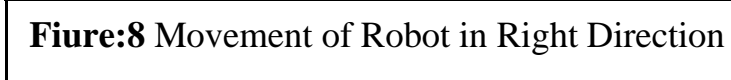

\begin{tabular}{|c|c|c|c|c|}
\hline $\begin{array}{c}\text { Sr. } \\
\text { No }\end{array}$ & $\begin{array}{c}\text { Decision } \\
\text { by Robot }\end{array}$ & $\begin{array}{c}\text { Sensor } \\
\text { Right }\end{array}$ & $\begin{array}{c}\text { Sensor } \\
\text { Straight }\end{array}$ & $\begin{array}{c}\text { Sensor } \\
\text { Left }\end{array}$ \\
\hline 1 & Straight & F & F & F \\
\hline 2 & Soft & F & F & C \\
\cline { 3 - 5 } 3 & Right & F & C & F \\
\cline { 3 - 5 } 4 & & F & C & C \\
\hline 5 & Hard & F & F & VC \\
\cline { 3 - 5 } 6 & Right & F & C & VC \\
\hline 7 & Soft & C & F & F \\
\cline { 3 - 5 } 8 & Left & C & C & F \\
\hline 9 & Hard & VC & F & F \\
\hline
\end{tabular}

Where; F-Far, C-Close, VC-Very Close

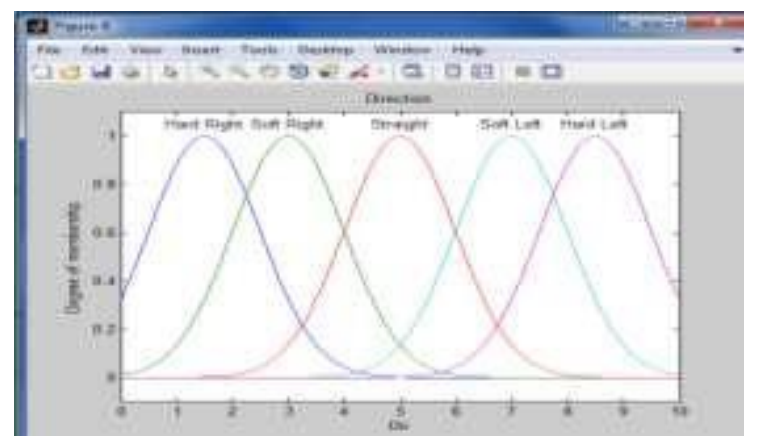

Figure: 9 Relation between degree of membership function and direction

All above figure shows relationship between membership function and distance.

\section{CONCLUSION}

In This paper we Proposed Adaptive fuzzy logic controller for finding out the collision free smooth path. Whereas simulation result shows the how the controller will find out the target.

\section{REFERENCES}

[1].Kaushik Das Sharma, AmitavaChatterjee, AndAnjanRakshit, “A PARTICAL SWARM OPTIMIZATION Lyapunov Hybrid Stable Adaptive Fuzzy Tracking Control Approach For Vision-Based Robot Navigation", IEEE TRANSACTIONS ON INSTRUMENTATION AND MEASUREMENT, VOL. 61, NO. 7, JULY 2012.

[2].Abdullah Zawawi MOHAMED \& Sang Heon LEE, "Autonomous Mobile Robot System Concept Based On PARTICAL SWARM OPTIMIZATION Path Planner and vSLAM", Hung Yao HSU Department of Advanced Manufacturing and Mechanical Engineering University of South Australia, South Australia, Australia.

[3].RamazanHavangi, Mohammad Ali Nekoui\& Mohammad Teshnehlab, "Adaptive Neuro-Fuzzy Extended Kalman 
Filtering for Robot Localization",Faculty of Electrical Engineering, K.N. Toosi University of Technology Tehran, IJCSI International Journal of Computer Science Issues, Vol. 7, Issue 2, No 2, March 2010.

[4].Fernando Rios-Gutierrez, and Christopher Jeanniton, “Qualitative Evaluation of a PID Controller for Autonomous Mobile Robot Navigation Implemented in an FPGA Card Rocio Alba-Flores", Dept. of Mechanical and Electrical EngineeringGeorgia Southern University, Statesboro, GA, USA 2011 Seventh International Conference on Natural Computation.

[5].Edward Tunstel, Tanya Lippincott and Mo Jamshidi, "Introduction to Fuzzy Logic Control With Application to Mobile Robotics", NASA Center for Autonomous Control Engineering, Department of Electrical and Computer EngineeringUniversity of New Mexico

[6]. “Decomposed Fuzzy Controller for Reactive Mobile Robot Navigation” International journal of soft computing and Engineering sept 2012.

[7].N. N. Singh, "Vision based autonomous navigation of mobile robots", Ph.D. dissertation, Jadavpur University, Kolkata, India, 2010.

[8].C. C. Chou, F. L. Lian, and C. C. Wang, "Characterizing indoor environment for robot navigation using velocity space approach with region analysis and look-ahead verification," IEEE Trans. Instrum. Meas., vol. 60, no. 2, pp. 442-451, Feb 2011.

[9].A. R. Ashokaraj, P. M. G. Silson, A. Tsourdos, and B.A. White, "Robust sensor-based navigation for mobile robots," IEEE Trans. Instrum. Meas., vol. 58, no. 3, pp. 551-556, Mar. 2010.

[10]. Jianguo, W., Yilong, Z., Linlin, X., Adaptive Genetic Algorithm Enhancements for Path Planning of Mobile Robots, Proceedings on International Conference on Measuring Technology and Mechatronics Automation 2010, pp. 416419.

[11]. J V. Gupta, K. Khare, and R. Singh, "Efficient FPGA Design and Implementation of Digital PID Controllers in Simulink", International Journal of Recent Trends in Engineering, 2(6), pp. 147- 150, Dec. 2009.

[12]. Hsu-Chih Huang, Department of Computer Science and Information Enginnering, Hungkuang and Ching-Chih, Tsai Department of Electrical Engineering, National Chung Hsing University, Taiwan "Global Path Planning for Autonomous Robot Navigation Using HybridMetaheuristic GA- PSO Algorithm” SICE Annual Conference 2011.

[13]. H. C. Huang and C. C. Tsai, "Simultaneous tracking and stabilization of anomni-directional mobile robot in polar coordinates: a unified control approach," Robotica, vol. 27, no. 3, pp. 447-458, May 2010.

[14]. William Low, R. Nagarajan, SazaliYaacob, "Simultaneous Localization and Map Building - A Guided Tour", Internationa Conference on Man-Machine Systems (ICoMMS), 11-13 October 2009 Penang, Malaysia.

[15]. S. C. Yun, S. Parasuraman, and V. Ganapathy, "Dynamic path planning algorithm in mobile robot navigation," Industrial Electronics and Applications (ISIEA), 2011 IEEE Symposium on, 2011. 